\title{
Special issue on multimedia networking for electronic commerce systems
}

\author{
Christos Grecos • Tasos Dagiuklas • \\ Martin Fleury • Dimitris Kanellopoulos
}

Published online: 13 February 2015

(C) Springer Science+Business Media New York 2015

\section{Introduction}

Multimedia networking applications benefit from the rapid development of encoding/compression techniques multimedia data sources, effective Quality of Service (QoS) management mechanisms and Quality of Experience (QoE) criteria for system optimization. This special issue aims to expose the readership to the latest research results on multimedia networking issues for electronic commerce systems.

The special issue is composed of three referred papers covering such topics as electronic souvenir appification (for smartphones and tablets), variable video coding for peer-to-peer (P2P) streaming, and effective P2P Internet Protocol television (IPTV) digital rights management architectures. The issue sets out to demonstrate pioneer work in this field, investigate novel solutions and methods for services design and discuss future trends in this field.

C. Grecos

Independent Imaging Consultant, Glasgow, UK

e-mail: grecoschristos@gmail.com

T. Dagiuklas

Hellenic Open University, Patras, Greece

e-mail: dagiuklas@eap.gr

M. Fleury

University of Essex, Colchester, UK

e-mail: fleum@essex.ac.uk

D. Kanellopoulos ( $\square)$

University of Patras, Patras, Greece

e-mail: d_kan2006@yahoo.gr 


\section{The papers in this special issue}

Recently, Appification has been introduced to describe the rapidly widening shift from web browsing to the usage of smartphone applications for Internet-based information access and e-services consumption. The first paper, "E-souvenir Appification: QoS Web based Media Delivery for Museum Apps" by Evangelos Sakkopoulos, Mersini Paschou, Yannis Panagis, Dimitris Kanellopoulos, Georgios Eftaxias and Athanasios Tsakalidis, presents a solution that introduces -souvenir (digital souvenir) by extending the appification of e-shopping and shopping (non electronic) souvenirs while visiting cultural sights (e.g. museums, exhibitions etc). The proposed smartphone app increases a user's involvement in order to personalize the App with his/her choices while sightseeing, and thus it forms an electronic souvenir. The proposed approach introduces intelligent QoS enabled features to the cultural smartphone App to improve the user's experience. In addition, the authors facilitate efficient QoS-based media delivery for the e-souvenir solution in order to make it efficient to operate under heavy network traffic load and best-effort Internet-based connections.

The second paper, "How Variable Bitrate Video Formats Can Help P2P Streaming Boost its Reliability and Scale" by Marat Zhanikeev, analyzes and compares performance among several P2P methods using simulation tools. Further, it presents a P2P delivery model that allows video content providers to run services globally without procuring local facilities in several countries. The paper also discusses the application of the proposed model in electronic commerce.

Currently, IPTV services are deployed in a P2P architecture to achieve scalability. In the light of this situation, the intellectual property rights of valuable IPTV content distributed over P2P networks must be considered. Definitely, an important issue is to effectively apply Digital Rights Management (DRM) for P2P IPTV services. The third paper, "A Cost-Effective Key Distribution of P2P IPTV DRM over Opportunistic Multicast Overlay for e-Commerce Systems" by Ray-I Chang, Tsao-Ta Wei, and Chia-Hui Wang, presents an effective P2P IPTV DRM architecture for e-Commerce systems. The proposed architecture is based on the standards from the open mobile alliance (OMA). In addition, the authors propose a cost-effective key distribution mechanism with leverage between DRM protection strength and system/network load. The proposed mechanism can improve DRM frameworks for P2P IPTV applications in e-Commerce systems on the opportunistic multicast overlay to overcome peer churn arising from leaving and joining peers.

Acknowledgments The guest editors wish to thank the Editor-in-Chief Prof. James Christopher. Westland, and the former EiC Prof. Bezalel Gavish for providing the opportunity to edit this special issue on Multimedia Networking for Electronic Commerce Systems, and other editors for providing latest publishing information and making this issue published. We would also like to thank the authors for submitting their works as well as the referees who have critically evaluated the papers. Finally, we hope the reader will share our joy and find this special issue very useful. 

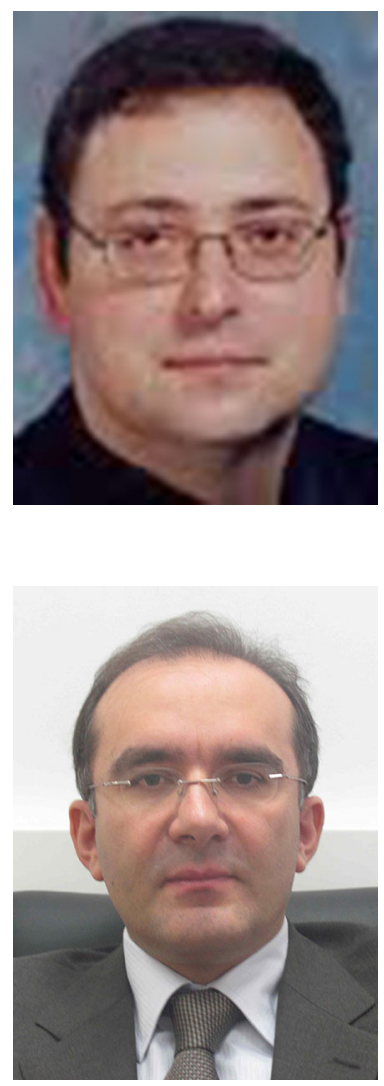

Christos Grecos is currently an Independent Imaging Consultant. Until the end of September 2014, he was a Professor in Visual Communications Standards, and Head of School of Computing, the University of the West of Scotland (UWS), United Kingdom (UK). He leaded the Audio-Visual Communications and Networks Research Group (AVCN) with UWS. His research interests include image/video compression standards, image/video processing and analysis, image/video networking and computer vision. He has published numerous research papers in top-tier international publications including a number of IEEE transactions on these topics. He is on the editorial board or served as guest editor for numerous international journals, and he has been invited to give talks in various international conferences. He was the Principal Investigator for several national or international projects funded by UK EPSRC or EU. He received his Ph.D. degree in Image/Video Coding Algorithms from the University of Glamorgan, UK.

Tasos Dagiuklas received the Engineering Degree from the University of Patras-Greece in 1989, the M.Sc. from the University of Manchester-UK in 1991 and the Ph.D. from the University of EssexUK in 1995, all in Electrical Engineering. He is Assistant Professor at the Hellenic Open University, School of Science \& Technology, Greece. He is the Research Leader of the CONES research group (http://cones.eap.gr). Past Positions include Faculty Position at the Department of Telecommunication Systems and Networks, TEI of Mesolonghi, and University of Aegean Greece. He has been involved in several EC R\&D Research Projects under FP5, FP6 and FP7 research frameworks as Principal Investigator. Dr Dagiuklas is Chair for IEEE MMTC 3DRPC IG and has served as Vice-Chair for IEEE MMTC QoE and Key Member of IEEE MMTC MSIG IGs. $\mathrm{He}$ is also an active member of IEEE P1907.1 Standardization WG. $\mathrm{He}$ is a reviewer for journals such as IEEE Transactions on Multimedia, IEEE Communication Letters and IEEE Journal on Selected Areas in Communications. He has served as TPC member to more than 50 international conferences and has served as Guest Editor for prestigious journals. His research interests include 2D/3D video transmission/adaptation/rate control across heterogeneous wireless networks, P2P video streaming, service provisioning across Future Internet architectures and Cloud infrastructures and services for media delivery. Dr Dagiuklas has published more than 130 papers at international journals, conferences and standardisation fora in the above fields. His research work has received more than 840 citations by other researchers. He is a Senior Member of IEEE and Technical Chamber of Greece.

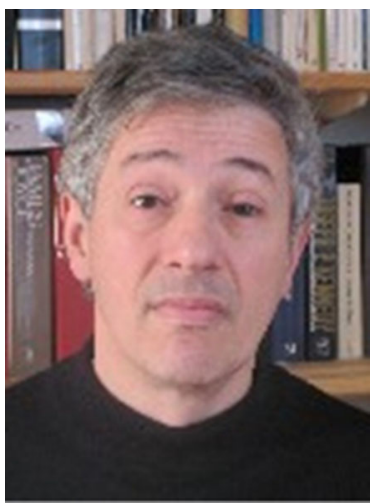

Martin Fleury received his degree in Modern History from Oxford University, UK and a Maths/Physics-based degree from the Open University, Milton Keynes, UK. He received his M.Sc. in Astrophysics from QMW College, University of London, UK in 1990 and M.Sc. from the University of South-West England, Bristol, UK in Parallel Computing Systems in 1991. He received a Ph.D. in Parallel Image Processing Systems from the University of Essex, Colchester, UK. He currently acts as a Visiting Fellow at the University of Essex, where he was previously employed as a Senior Lecturer, and where he was the Director of Graduates (Research). He is an External Examiner for the Arab Open University. He has authored around 250 articles, book chapters and a book on high-performance computing for low-level image- and signal-processing algorithms. His current research interests centre upon video communication over wired and wireless networks. 


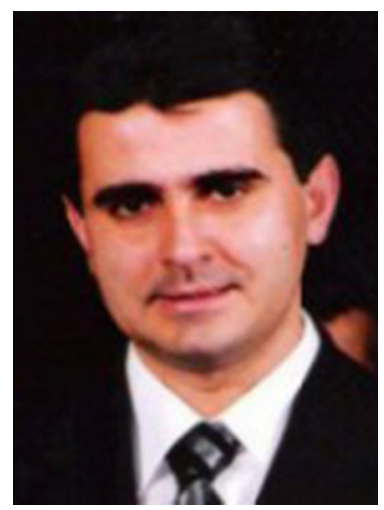

Dimitris Kanellopoulos is a member of the Educational Software Development Laboratory (ESDLab) in the Department of Mathematics at the University of Patras, Greece. He received the Electrical Engineering Degree and the Ph.D. in multimedia communications from the University of Patras. Since 1990, he was a research assistant in the Department of Electrical and Computer Engineering at the University of Patras and involved in several EU R\&D projects. His current research interests include multimedia communications, image/video networking, intelligent information systems, semantic web, and knowledge representation. Recently, he edited a book: "Intelligent Multimedia Technologies for Networking Applications: Techniques and Tools", while a "Handbook of Research on Networked Multimedia Communication Systems" is under preparation. He is a member of the IEEE Multimedia Communications Technical Committee and he has many publications to his credit in international journals and conferences at these areas. He has served as TPC member to more than 40 international conferences and has served as Guest Editor for prestigious journals. 